\title{
Neue rechtliche Möglichkeiten für die Zertifizierung
}

\author{
Undine Soltau ${ }^{a, b} \quad$ Carsten Sterly \\ a Zentralstelle der Länder für Gesundheitsschutz bei Arzneimitteln und Medizinprodukten, Bonn, \\ ${ }^{b}$ Fachbeirat Gesundheit und Forensik des Akkreditierungsbeirates im Bundesministerium für Wirtschaft und Technologie (BMWi), \\ Berlin, Deutschland
}

Seit 2004 sind deutsche Vertragsärzte, medizinische Versorgungszentren und Krankenhäuser verpflichtet, ein Qualitätsmanagement einzuführen und weiterzuentwickeln (§ 135 SGB V). Es wird dafür jedoch kein einheitliches Zertifizierungsverfahren genutzt. Verschiedene Systeme wie KTQ (Kooperation für Transparenz und Qualität im Gesundheitswesen) oder EFQM (European Foundation for Quality Management) und DIN EN ISO 9001 kommen zur Anwendung. Die Zertifizierung erfolgt in der Regel durch unabhängige Dritte, meist privatwirtschaftliche Zertifizierungsunternehmen. Deren Akkreditierung ist nicht zwingend. Tatsächlich beschränken sich diese Akkreditierungen derzeit auf Stellen, die Qualitätsmanagement-Systeme nach DIN EN ISO 9001 zertifizieren.

Prinzipiell bestätigt ein Zertifikat lediglich, dass die geprüfte Einrichtung nach einer bestimmten Norm beziehungsweise einem vorgegebenen normativen Katalog handelt. Sie bewegt sich damit innerhalb eines definierten Organisationsrahmens, der aber je nach gewähltem Zertifizierungsschema mit sehr unterschiedlichen Inhalten gefüllt ist. So kann die Verleihung eines Zertifikats eine mehrtägige, aber auch eine mehrwöchige Fortbildung voraussetzen; der Schwerpunkt beim Zertifizieren kann auf dem leitlinienkonformen Handeln liegen oder Parameter wie Personenkompetenz und Geräteausstattung stärker berücksichtigen. Daraus resultiert eine Vielzahl von Zertifikaten, die nur bedingt vergleichbare Aussagen zur Qualität zulassen. Wird eine Zertifizierung angestrebt, klärt die Einrichtung vorab, welches Zertifikat sich für die jeweilige Zielsetzung am besten eignet. Schließlich soll damit eine größtmögliche Anerkennung bei der jeweiligen Zielgruppe - dem Patienten, dem Arbeitgeber, dem Leistungsträger, der Behörde usw. - erreicht werden.

\section{Akkreditierung - eine Maßnahme zur Vertrauensbildung}

Akkreditierung bezweckt, den Inhalt und das Niveau von Zertifikaten und Prüfberichten auf eine einheitliche, dem internationalen Stand entsprechende Basis zu stellen. Der Europäische Rat und das Parlament haben einen neuen, für alle Mitgliedstaaten verbindlichen Rechtsrahmen geschaffen, um dieses Ziel wirksamer als in der Vergangenheit durchzusetzen. Akkreditierung im Sinne der neuen Regelungen wird in allen Wirtschafsbereichen Anwendung finden. Zunehmend ersetzen Zertifikate akkreditierter Stellen sogar behördliche Zulassungen.

Akkreditierung ist in der Verordnung (EG) 765/2008 definiert als «die Bestätigung durch eine nationale Akkreditierungsstelle, dass eine Konformitätsbewertungsstelle [z.B. eine Zertifizierungsstelle] die in harmonisierten Normen festgelegten Anforderungen und gegebenenfalls zusätzliche Anforderungen, einschließlich solcher in relevanten sektoralen Akkreditierungssystemen erfüllt, um eine spezielle Konformitätsbewertungstätigkeit durchzuführen».

$\mathrm{Neu}$ ist daran, dass die normativen Anforderungen (z.B. DIN EN ISO 17024 für Personen-Zertifizierungsstellen und DIN EN ISO 17021 für Qualitätsmanagement-Zertifizierungsstellen) durch sektorale Akkreditierungsregeln untersetzt werden sollen, die den jeweiligen fachspezifischen Anforderungen Rechnung tragen.

Näheres dazu findet sich im New Legislative Framework (NLF) bestehend aus den Verordnungen (EG) 764/2008 [1], (EG) 765/2008 [2] sowie dem Beschluss 768/2008/EG [3] und im Akkreditierungsstellengesetz (AkkStelleG).

Sektorale Akkreditierungsregeln sollen künftig nach den gesetzlichen Vorgaben ermittelt und über die European cooperation for Accreditation (EA, www.european-accreditation. org) europäisch harmonisiert werden. Nationale Behörden

\begin{tabular}{ll}
\hline KARGER & ( 2012 S. Karger GmbH, Freiburg \\
0378-584X/12/3516-0007\$38.00/0 \\
$\begin{array}{l}\text { Fax+49 7614520714 } \\
\text { Information@Karger.de } \\
\text { www.karger.com }\end{array}$ & $\begin{array}{l}\text { Accessible online at: } \\
\text { www.karger.com/onk }\end{array}$
\end{tabular}




\section{Vertrauensbildung}

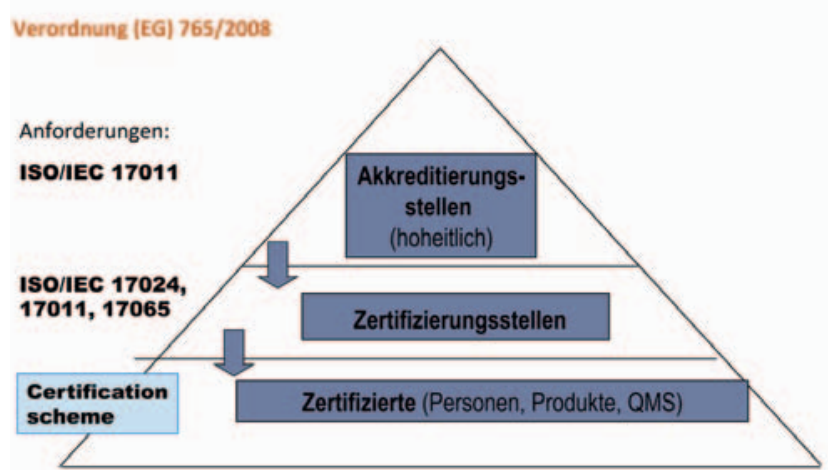

Abb. 1. Rahmenbedingungen für eine Zertifizierung.

werden durch die oben genannten Verordnungen verpflichtet, «die Gleichwertigkeit der von den Akkreditierungsstellen erbrachten Dienstleistungen anzuerkennen und damit die Akkreditierungsurkunden und die Bestätigungen, die von den von ihnen akkreditierten Konformitätsbewertungsstellen ausgestellt wurden, zu akzeptieren». Das heißt, Behörden und Kostenträger sind verpflichtet, Zertifikate und Prüfberichte akkreditierter Stellen ohne weitere Auflagen anzuerkennen, sofern dem nicht andere Gesetze mit spezielleren Anforderungen (z.B. das Medizinproduktegesetz) entgegenstehen.

Jeder EU-Mitgliedsstaat hat gemäß den europäischen Vorgaben jeweils eine Stelle eingerichtet, die im Auftrag des Staates und damit hoheitlich handelnd Akkreditierungen durchführt (Abb. 1). In Deutschland ist es die Deutsche Akkreditierungsstelle GmbH (DAkkS) mit Sitz in Berlin. Aufgrund des Beschlusses 768/2008/EG zur Schaffung eines gemeinsamen Rechtsrahmens in Fragen der Akkreditierung und Marktüberwachung werden die künftigen europäischen Rechtsakte diesem Ansatz folgen und Akkreditierung als vertrauensbildende Maßnahme fördern. Die Erwägungsgründe, die zur Verabschiedung der Verordnung (EG) 765/2008 über Akkreditierung und Marktüberwachung führten, waren vielfältig. So war die Akkreditierung bis dato nicht auf Gemeinschaftsebene geregelt, wurde aber dennoch in allen Mitgliedsstaaten praktiziert. Dies führte zu unterschiedlichen Systemen sowie zu mehr oder weniger strenger Handhabung der Akkreditierungsanforderungen.

Im Hinblick auf die zunehmende grenzüberschreitende Gesundheitsversorgung muss infolge der neuen Rechtslage eine Vergleichbarkeit der Akkreditierungen und damit auch der akkreditierten Prüfungen und Zertifizierungen erreicht werden.

Die neuen Regelungen sind europaweit rechtsverbindlich. Sie bieten neue Chancen, akkreditierten Zertifizierungsstellen, Laboratorien und Inspektionsstellen, aber auch Personen, Zertifikaten und Prüfberichten die gewünschte Anerken- nung zu verschaffen. Der derzeit fragliche Wert vieler Zertifikate kann deutlich gestärkt werden, wenn es gelingt, sektorale Akkreditierungsregeln und Zertifizierungsprogramme unter Einbeziehung der Kreise zu erarbeiten, an die sich die Zertifikate richten.

\section{Wer kann sich akkreditieren lassen?}

Das Angebot der Zertifizierungsstellen kann die Zertifizierung von Produkten (DIN EN ISO/IEC 17065), von Managementsystemen (DIN EN ISO/IEC 17021) oder Personen (DIN EN ISO/IEC 17024) umfassen. Zum Zwecke der Akkreditierung müssen die in den genannten, weltweit geltenden Normen festgelegten Anforderungen erfüllt sein.

\section{Zertifizierung von Personen}

Die Zertifizierung von im medizinischen Bereich tätigen Personen ist ein relativ neues und derzeit aktiv bearbeitetes Feld, das deshalb an dieser Stelle näher beleuchtet werden soll.

Personen-Zertifizierungen sind in anderen Bereichen bereits seit langem etabliert (z.B. Qualitätsmanagement-Personal, IT-Spezialisten, Immobilien-, KFZ- und sonstige Sachverständige), im Gesundheitswesen jedoch noch selten. Derzeit gibt es lediglich ein Zertifizierungsverfahren für Personen, die auf dem Gebiet der Wundversorgung arbeiten. Es basiert auf einem Curriculum der Deutschen Gesellschaft für Wundheilung und Wundbehandlung e.V. sowie der Akkreditierung einer Zertifizierungsstelle auf Basis der DIN EN ISO/IEC 17024 für diesen Geltungsbereich. Einer als Wundpfleger/ Wundtherapeut zertifizierten Person wird Fachkompetenz nach bundeseinheitlichen, leitlinienbasierten Kriterien bestätigt. Innerhalb der letzten 3 Jahre haben mehr als 1000 Antragsteller dieses Zertifizierungsverfahren erfolgreich durchlaufen. Insgesamt wird das Angebot jedoch noch zu selten genutzt, was in Zusammenhang mit dem Aufwand für die Zertifizierung zu sehen ist. Die Zertifizierung als Wundpfleger/Wundtherapeut erfordert den Nachweis von Erfahrungen und Kenntnissen, die in einem Grundkurs über 84 Stunden und einem Aufbaukurs über 286 Stunden vertieft werden.

Weitere Personen-Zertifizierungen im Gesundheitswesen erfordern die Bereitstellung entsprechender Curricula, die im Detail auf ihre Eignung für Zertifizierungszwecke (Zertifizierungsprogramm/Certification Scheme) geprüft werden und sich zunächst im Rahmen von Pilotprojekten bewähren müssen. Entsprechende Ansätze gibt es zum Beispiel im Bereich der Stoma- und Ernährungstherapie. Akkreditierte PersonenZertifizierungen auf Basis von DIN EN ISO/IEC 17024 sollen künftig auch in Tarifverträge Eingang finden und damit die Motivation zur Teilnahme an einem Zertifizierungsverfahren steigern.

Neue Zertifizierungen im medizinischen bzw. pflegerischen Bereich können ein wichtiger Beitrag zum Nachweis und Erhalt von Kompetenz sein und künftig auch eine we- 


\section{Gremien}

\section{Akkreditierungsbeirat des $\mathrm{BMWi}$}

\section{Fachbeiräte}

\begin{tabular}{|ll|}
\hline 1 & Bauwesen / Verkehr / Werkstofftechnik / Materialprüfung \\
\hline 2 & Geräte- und Anlagensicherheit / EMV / Telekommunikation \\
\hline 3 & Gesundheit, Forensik \\
\hline 4.1 & Gesundheitlicher Verbraucherschutz / Agrar \\
4.2 & Chemie / Umwelt \\
\hline 5 & Metrologie \\
\hline 6 & System- / Personenzertifizierung \\
\hline 7 & Horizontale Fragen \\
\hline
\end{tabular}

Abb. 2. Fachbeiräte im Akkreditierungsbeirat des BMWi.

\section{AkkStelle $\bigcirc$ Regelermittlung}

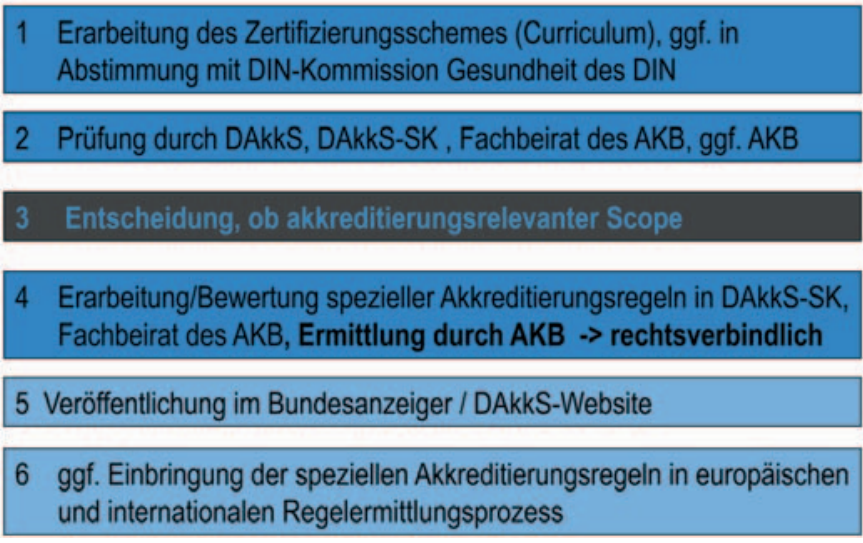

Abb. 3. Regelermittlung im Akkreditierungsbeirat.

\section{AkkStelle $\bigcirc$ CHANCE}

\begin{tabular}{|l}
\hline $\begin{array}{r}\text { Leistungsträger, Behörden und andere Zielgruppen in den Fachbeirat } 3 \\
\text { Gesundheit/Forensik einbinden und an der Regelermittlung beteiligen }\end{array}$ \\
\begin{tabular}{|l}
\hline 2 Regeln mit Anforderungen an zu Zertifizierende \\
an Zertifizierungsstelle \\
an Akkreditierung der Zertifizierungsstelle
\end{tabular} \\
\begin{tabular}{|l} 
4. Verbindlichkeit der Regeln durch Ermittlung im Akkreditierungsbeirat und \\
Veröffentlichung im Bundesanzeiger / auf DAkkS-Website
\end{tabular} \\
\begin{tabular}{|l} 
5. Akzeptanz der Zertifizierungen akkreditierter Zertifizierer \\
durch die Zielgruppe
\end{tabular} \\
\hline 6 Regeln ggf. europäische/internationaler Anerkennung zuführen \\
\hline
\end{tabular}

Abb. 4. Chance aufgrund des Akkreditierungsstellengesetzes. sentliche Funktion im Bereich der integrierten Versorgung haben. Dabei sollten neue Zertifizierungsvorhaben stets auch die Akkreditierung im Auge haben, um als zukunftsfähige Konzepte im Wettbewerb bestehen zu können. Neue, von der deutschen Akkreditierungsstelle anerkannte Personen-Zertifizierungen werden in Verzeichnisse aufgenommen und sind über die DAkkS-Homepage (www.dakks.de) einsehbar.

Die Norm DIN EN ISO/IEC 17024 verlangt, dass Zertifizierungsprogramme für Personen nur aufgrund von spezifischen öffentlich-rechtlichen Anforderungen (z.B. zum Schutz der Öffentlichkeit) oder aufgrund eines nachgewiesenen Bedarfs/Wunsches des Marktes (im Besonderen im Hinblick auf Glaubwürdigkeit, Vertrauen und Verbesserung des Berufsbildes) eingerichtet werden. Im Falle des Wundpflegers bzw. -therapeuten handelt es sich um eine «gefahrgeneigte Tätigkeit», weshalb für das Zertifizierungsverfahren auch eine Durchfallquote vereinbart wurde, um damit Anspruch und Qualität der Zertifizierung hervorzuheben. Zertifizierungen werden in der Regel berufsübergreifend angeboten und können somit - insbesondere für Subspezialisierungen, für die keine entsprechende Facharztqualifizierung möglich ist auch von Ärzten avisiert werden.

Die Organisation oder die Zertifizierungsstelle, die das Zertifizierungsprogramm vorschlägt, sollte die Betroffenen und die in die Zertifizierung involvierten Kreise konsultieren: - zwecks Beschreibung des spezifischen Bereichs, für den die Personen zertifiziert werden sollen (SCOPE);

- zwecks Beschreibung der Qualifikations-/Kompetenzanforderungen, Bewertungsanforderungen und -verfahren, einschließlich derer für die Überwachung und Rezertifizierung;

- bezüglich des Grades der Unterstützung für das Programm durch die interessierten Kreise und die Nachweise der Akzeptanz von Inhalten des Programms;

- zur Klärung der Frage, welche Organisation/Stelle oder Person für die Entwicklung des vorgeschlagenen Programms verantwortlich sein soll.

Ein Zertifizierungsschema für Personen nach DIN EN ISO/ IEC 17024 sieht ferner eine Arbeits- bzw. Praxisanalyse nach jeweils 5 Jahren vor. Diese muss unter anderem eine Aussage über Zweck und Auswirkung der Zertifizierung sowie Angaben zu den Zertifizierungsanforderungen, den Auswertungsmechanismen und zum Aufbau der Prüfungen enthalten.

\section{Die Aufgaben des Fachbeirates Gesundheit/Forensik des Akkreditierungsbeirates nach $\$ 5$ AkkStelleG}

Gemäß Akkreditierungsstellengesetz wurde vom Bundesministerium für Wirtschaft und Technologie (BMWi) im Einvernehmen mit 7 fachlich betroffenen Bundesministerien (Bundesministerium für Gesundheit; Arbeit und Soziales; Ernährung, Landwirtschaft und Verbraucherschutz; Verkehr, Bau und Stadtentwicklung; Umwelt, Naturschutz und Reaktorsi- 
cherheit; Finanzen; und Bundesministerium des Innern) ein ausgewogen $\mathrm{zu}$ besetzender Akkreditierungsbeirat berufen (www.bmwi.de, www.akb.bam.de). $\mathrm{Zu}$ dessen gesetzlichen Aufgaben gehört die Einrichtung von Fachbeiräten (Abb. 2-4). Die 7 Fachbeiräte widmen sich den jeweiligen Zertifizierungsbereichen und beraten den Akkreditierungsbeirat in Fachfragen. Entscheidungen des Akkreditierungsbeirates sowie «ermittelte» Akkreditierungsregeln werden nach $\mathrm{Zu}$ stimmung des fachlich betroffenen Bundesministeriums (siehe oben) im Bundesanzeiger und auf der DAkkS-Website veröffentlicht.

Ein aktuelles, in den Fachbeiräten 3 und 6 behandeltes Projekt betrifft die «Zertifizierung von betrieblichen Gesundheitsmanagementsystemen».

Aufgrund der umfangreichen Vorarbeiten auf dem Gebiet der Personen-Zertifizierung wird die Etablierung weiterer Zertifizierungen künftig einfacher. Der Aufwand für interessierte Fachgesellschaften, Zertifizierungsprogramme innerhalb ihres Fachgebietes auszuarbeiten, hält sich im Rahmen. Da die Regeln für die Akkreditierung auch international vermehrt Anerkennung finden, ist deren Berücksichtung für künftige Zertifizierungsvorhaben unbedingt wünschenswert.

Der neue Rechtsrahmen bietet die Möglichkeit, aufgrund verbindlicher Vorgaben vergleichbare, qualitativ hochwertige, den Anforderungen der Zielgruppen genügende Zertifikate und Anerkennungen zu generieren. Dem Dilemma, dass teuren, aber nicht wirklich vergleichbaren Zertifikaten die Akzeptanz verwehrt wird, kann nur entgegengewirkt werden, wenn die neuen, aus dem AkkStelleG resultierenden Chancen vollumfänglich genutzt werden.

\section{Disclosure Statement}

Es bestehen keine Interessenkonflikte.

\section{Literatur}

1 Regulation (EC) No $765 / 2008$ of the European Parliament and of the Council of 9 July 2008 setting out the requirements for accreditation and market surveillance relating to the marketing of products and repealing Regulation (EEC) No $339 / 93$.
2 Regulation (EC) No 764/2008 of the European Parliament and of the Council of 9 July 2008 laying down procedures relating to the application of certain national technical rules to products lawfully marketed in another Member State and repealing Decision No 3052/95/EC.
3 Decision No 768/2008/EC of the European Parliament and of the Council of 9 July 2008 on a common framework for the marketing of products, and repealing Council Decision 93/465/EEC. 